Bull. Austral. Math. Soc.

$11 \mathrm{G} 09,11 \mathrm{~J} 85,11 \mathrm{~J} 93$

VoL. $74(2006)$ [461-470]

\title{
INDÉPENDANCE ALGÉBRIQUE DE LOGARITHMES EN CARACTÉRISTIQUE $P$
}

\section{LAURENT DENIS}

\begin{abstract}
Let $k$ be the rational function field over the field with $q$ elements with characteristic $p$. Since the work of Carlitz we know in this situation the function $\zeta$ analog of the Riemann zeta function and the function $\log _{\phi}$ analog of the usual logarithm. We will show two main results. Firstly, if $\xi$ denotes the fundamental period of Carlitz module, we prove that $\xi, \zeta(1), \ldots, \zeta(p-2)$ are algebraically independent over $k$. Secondly if $a_{1}, \ldots, a_{n}$ are rational elements (of degree less than $q /(q-1)$ to ensure convergence of the logarithm) such that $\log _{\phi} a_{1}, \ldots, \log _{\phi} a_{n}$ are linearly independent over $k$ then they are algebraically independent over $k$. The point is to find suitable functions taking these values and for which Mahler's method can be used.
\end{abstract}

\section{RÉSUltats ET SITUATION}

Soit $A=F_{q}[T]$ l'anneau des polynômes à coefficients dans le corps à $q$ éléments $F_{q}$ dont on note $p$ la caractéristique et $k=F_{q}(T)$ son corps des fractions. L'opposé du degré munit $k$ d'une valuation $v=-\operatorname{deg}$, on notera $k_{\infty}=F_{q}((1 / T))$ le complété de $k$ pour cette valuation. La valuation $v$ se prolonge de manière unique à la clôture algébrique $\overline{k_{\infty}}$ de $k$ et à son complété $C$ qui reste un corps algébriquement clos. On notera $|x|=q^{\operatorname{deg}(x)}$ la valeur absolue d'un élément $x$ de $C$ avec la convention $\operatorname{deg}(0)=-\infty$. On désigne aussi par $\bar{k}$ le corps des éléments de $C$ qui sont algébriques sur $k$ et on les appellera plus simplement nombres algébriques.

DÉfinition 1.1: Le module de Carlitz est la donnée du groupe additif $G_{a}$ et de l'homomorphisme $F_{q}$-linéaire et injectif d'anneaux $\Phi: A \rightarrow \operatorname{End}\left(G_{a}\right)$ défini par:

$$
\phi(T)=T F^{0}+F,
$$

où $F$ est le Frobenius relatif à $q$.

Il existe alors une unique fonction exponentielle $e_{\phi}$ caractérisée par les propriétés:

$$
\begin{aligned}
e_{\phi}(0) & =0, d / d z\left(e_{\phi}(z)\right)=1 \\
\text { pour tout } z \in C: e_{\phi}(T z) & =\phi(T)\left(e_{\phi}(z)\right)=T e_{\phi}(z)+e_{\phi}(z)^{q} .
\end{aligned}
$$

Received 27th June, 2006

Copyright Clearance Centre, Inc. Serial-fee code: 0004-9727/06 \$A2.00+0.00. 
Rappelons (voir [3]) que cette exponentielle est $F_{q}$-linéaire, qu'il existe un élément $\pi$ de $k_{\infty}$ tel que ker $e_{\phi}=A\left(T-T^{q}\right)^{1 /(q-1)} \pi$ et qu'elle admet une bijection réciproque dans le disque $\operatorname{deg}(z)<q /(q-1)$ noté $\log _{\phi}$.

DÉfinıtion 1.2: Pour tout entier naturel $h>0$, on pose: $[h]=T^{q^{h}}-T$ et $[0]=1$ et on définit par récurrence les suites $D_{h}$ et $L_{h}$ par $D_{0}=1=L_{0}$ et pour $h \geqslant 1$, $D_{h}=[h]\left(D_{h-1}\right)^{q}$ et $L_{h}=[h] L_{h-1}$.

L'équation fonctionnelle de l'exponentielle conduit alors à (voir [3]):

$$
e_{\phi}(z)=\sum_{0 \leqslant h \leqslant \infty}\left(z^{q^{h}} / D_{h}\right)
$$

pour tout $z$ dans $C$ et $\log _{\phi}(z)=\sum_{0 \leqslant h \leqslant \infty}\left((-1)^{h} z^{q^{h}}\right) / L_{h}$ pour tout $z$ de degré $<q /(q-1)$.

Rappelons enfin que la fonction zéta de Carlitz, analogue de la fonction zéta usuelle est définie pour tout $s$ entier naturel $>0$ par: $\zeta(s)=\sum_{a \in A+} 1 / a^{s}$ (où $A+$ désigne l'ensemble des éléments unitaires de $\mathrm{A}$ ) et qu'elle vérifie pour $1 \leqslant s \leqslant p$,

$$
\zeta(s)=\sum_{0 \leqslant h \leqslant \infty}\left((-1)^{h s} /\left(L_{h}\right)^{s}\right)
$$

(voir [3]).

Les derniers résultats connus sur la transcendance des valeurs de la fonction zéta sont obtenus en 1997 par Yu (voir [13]). Il démontre que les seules relations de dépendance linéaire sur $\bar{k}$ entre les $\pi^{s}(0 \leqslant s \leqslant \infty)$ et les $\zeta(s)(1 \leqslant s \leqslant \infty)$ proviennent des relations $\zeta(s) /(\pi)^{s} \in k$ pour $s$ multiple de $p-1$. Notons aussi qu'en 1994 de Mathan (voir [10]) a prouvé que certains polynômes (de degrés petits) à $p-1$ variables évalués en $(\zeta(1), \ldots, \zeta(p-2), 1 / \pi)$ étaient transcendants. Nous allons prouver ici le théorème suivant:

THÉORÈme 1.3. Les $p-1$ nombres $\zeta(s)(1 \leqslant s \leqslant p-1)$ sont algébriquement indépendants sur $k$.

REMARQUES. (a) Le Théorème 1 revient à dire que $\pi$ et les $\zeta(s)(1 \leqslant s \leqslant p-2)$ sont algébriquement indépendants sur $k$.

(b) La limitation aux valeurs $\zeta(s)$ pour $(1 \leqslant s \leqslant p-1)$ est légitime puisque $\zeta(p)$ $=\zeta(1)^{p}$. Cependant le fait que la formule

$$
\zeta(s)=\sum_{0 \leqslant h \leqslant \infty}\left((-1)^{h s}\right) /\left(\left(L_{h}\right)^{s}\right)
$$

ne s'étende pas aux valeurs de $s>p$ ne nous a pas permis d'obtenir le degré de transcendance du corps faisant intervenir ces valeurs (remarquons qu'une extension de cette formule au cas $s>p$, a été établie par Damamme et Hellegouarch (formule (48) de [9]) mais qu'elle comporte des termes en $D_{h}$ ce qui nous semble interdire l'usage de notre méthode). 
Pour ce qui est des valeurs du logarithme en des points algébriques, on connait l'analogue du théorème de Baker qui affirme l'indépendance linéaire sur $\bar{k}$ de 1 et de logarithmes de nombres algébriques linéairement indépendants sur $k$ (voir $[4,5,14]$ ou [13] pour des preuves différentes).

Nous allons établir l'énoncé suivant:

THÉORÈME 1.4. Soit $e$ un entier naturel non nul et $\alpha_{1}, \ldots, \alpha_{n}$ des éléments de $F_{q}\left(T^{1 / e}\right)$ qu'on suppose tous de degré $<q /(q-1)$.

Si $\log _{\phi}\left(\alpha_{1}\right), \ldots, \log _{\phi}\left(\alpha_{n}\right)$ sont linéairement indépendants sur $k$ alors ils sont algébriquement indépendants sur $\bar{k}$.

Remarque. Il s'agit donc d'une version un peu plus générale que le "cas rationnel" de la conjecture d'indépendance algébrique des logarithmes pour le module de Carlitz.

Contrairement aux résultats précédemment cités sur ces questions qui utilisaient la théorie de la transcendance sur les t-modules, la méthode consiste ici à trouver des fonctions prenant les valeurs qui nous intéressent en des points algébriques et vérifiant des équations fonctionnelles de type Malher. Nous avions déjà utilisé cette idée dans le cas beaucoup plus particulier de $\pi$ et de ses dérivées dans $[6,7]$.

Dans le paragraphe suivant, nous introduisons quelques fonctions satisfaisant des équations fonctionnelles et établissons des conditions d'indépendance algébrique de ces fonctions. Enfin, dans la troisième partie, nous en déduisons les Théorèmes 1.3 et 1.4 ainsi que quelques autres énoncés d'indépendance algébrique.

\section{INDÉPENDANCE DE FONCTIONS}

Commençons par introduire les fonctions qui vont nous servir et à établir les premières propriétés qu'elles vérifient.

Soit $\alpha(z)$ un élément non nul de $F_{q}((1 / z))$, dont on note $\operatorname{deg}_{z}(\alpha)$ son degré en la variable $z$, a un élément de $\bar{k}$ non nul et $g$ le polynôme défini par $g(z)=z^{e q}-T$ où $e$ est un entier fixé. On considère alors pour tout entier naturel $s>0$, l'expression formelle (où on utilise la convention qu'un produit vide vaut 1 ):

$$
f(a, \alpha, s)(z)=\sum_{0 \leqslant h \leqslant \infty} \frac{a^{h} \alpha(z)^{q^{h}}}{\left[g(z) \ldots g\left(z^{q^{h-1}}\right)\right]^{s}} .
$$

LEMME 2.1.

(a) Si $\operatorname{deg}_{z}(\alpha)<\operatorname{seq} /(q-1)$, l'expression $f(a, \alpha, s)(z)$ définit une fonction entière dans le domaine de $C$ délimité par la condition $\operatorname{deg}(z)$ $>1 /(q e)$.

(b) Sous les conditions précédentes, les coefficients du développement en $1 / z$ de $f(a, \alpha, s)$ appartiennent à $k(a)$ et on a l'équation fonctionnelle: 


$$
f(a, \alpha, s)\left(z^{q}\right)=\left(g(z)^{s} / a\right)[f(a, \alpha, s)(z)-\alpha(z)] .
$$

Preuve: (a) et (b) pour $z$ dans $C$ de degré $>1 /(q e)$, le degré du terme général de la somme est égal à $h \operatorname{deg}(a)+q^{h} \operatorname{deg}_{z}(\alpha) \operatorname{deg}(z)-s e\left(q+\cdots+q^{h}\right) \operatorname{deg}(z)$ qui tend bien vers $-\infty$ quand $h$ tend vers $+\infty$. On peut ensuite utiliser le développement de $\alpha(z)$ dans $F_{q}((1 / z)$ ) (qui converge puisque $\operatorname{deg}(z)>0$ ) pour s'apercevoir que pour chaque entier $m$, seuls un nombre fini de termes généraux de la somme apportent un coefficient en $1 / z^{m}$. Enfin l'équation fonctionnelle provient du fait que $\alpha\left(z^{q}\right)=[\alpha(z)]^{q}$ puisque les coefficients de $\alpha(z)$ sont dans $F_{q}$.

LEMME 2.2. Si $\alpha(z)$ est une fonction algébrique sur $C(z)$ alors $h(z):=f(a, \alpha, s)(z)$ est une fonction transcendante sur $C(z)$.

PREUVE: Comme $\alpha(z)$ est dans $F_{q}((1 / z))$, pour $i$ entier naturel, $T^{1 / e^{i}}$ n'est pas $^{\prime}$ un pôle de $\alpha(z)$. Pour tout tel $i$, la somme $h_{i}(z)$ des $i$ premiers termes de l'expression de $h(z)$ (qui appartient à $C(z)$ ) n'a pas non plus de pôle en $T^{1 / e q^{i}}$. Comme le produit $g(z) \ldots g\left(z^{q^{i}}\right)$ est dans le dénominateur de tous les termes suivants, $h(z)-h_{i}(z)$ a un pôle en $T^{1 / e^{i}}$ sauf si c'est la fonction nulle. Donc $h(z)$ qui par la discussion précédente se prolonge en une fonction méromorphe dans $\operatorname{deg}(z)>0$, a une infinité de pôles et est donc une fonction transcendante sur $C(z)$, pourvu que pour une infinité d'entiers $h(z)-h_{i}(z)$ soit non nulle. Or $h(z)-h_{i}(z)$ n'est pas nulle pour $i$ assez grand puisque le degré du terme général est strictement décroissant pour $\operatorname{deg}(z)$ assez grand (voir le calcul explicite du Lemme 2.1).

Passons maintenant aux propriétés d'indépendance de certaines de ces fonctions. Dans la suite de ce texte, nous devrons nous restreindre au cas où $\alpha(z)$ est une fraction rationnelle non nulle appartenant à $F_{q}(z)$. Nous utiliserons le lemme suivant:

LEMME 2.3. Soit $L(X)$ un polynôme à coefficients dans $C$ ayant pour seule racine dans $C$ un élément a qui n'est pas dans $\overline{F_{q}}$ (la clôture algébrique de $F_{q}$ ). Si $f$ appartient à $C(X)$ et vérifie: $f\left(X^{q}\right) L(X)=f(X)$ alors $f$ est nulle.

PreUve: Si $z$ est un pôle de $f$ alors pour tout entier $n, z^{q^{n}}$ est un pôle de $f$ donc $z$ appartient à $\overline{F_{q}}$. Donc $a$ est un zéro de $f$ et de proche en proche pour tout $n, a^{1 / q^{n}}$ également. Donc $f$ est nulle.

Donnons un énoncé d'indépendance où l'important est que le " $s$ " de $f(a, \alpha, s)$ varie:

Lemme 2.4. Etant donné $\alpha_{1}(z), \ldots, \alpha_{p-1}(z), p-1$ éléments non nuls de $F_{q}(z)$ vérifiant $\operatorname{deg}_{z}\left(\alpha_{i}\right)<i e q /(q-1)(1 \leqslant i \leqslant p-1), a_{1}, \ldots, a_{p-1}, p-1$ éléments non nuls de $\bar{k}$, les fonctions $h_{i}(z):=f\left(a_{i}, \alpha_{i}, i\right)(z)$ sont algébriquement indépendantes sur $C(z)$.

Preuve: On montre l'énoncé par récurrence sur l'entier $j \leqslant p-1$. La propriété est vraie pour $j=1$ par le Lemme 2.2. D'après l'hypothèse de récurrence si $h_{1}(z), \ldots, h_{j}(z)$ sont algébriquement dépendantes sur $C(z)$ il existe un polynôme irréductible $P \in$ $C\left[X_{0}, X_{1}, \ldots, X_{j}\right]-C\left[X_{0}\right]$ engendrant l'idéal des relations de dépendances des fonctions 
sur $C$ tel que pour tout $z$ dans le domaine de convergence on ait:

$$
P\left(z, h_{1}(z), \ldots, h_{j}(z)\right)=0,
$$

et donc aussi:

$$
P\left(z^{q},\left(g(z) / a_{1}\right)\left[h_{1}(z)-\alpha_{1}(z)\right], \ldots,\left(g(z)^{j} / a_{j}\right)\left[h_{j}(z)-\alpha_{j}(z)\right]\right)=0 .
$$

Quitte à multiplier cette relation par une puissance convenable d'un dénominateur commun aux $\alpha_{i}(z)$, on voit qu'il existe $R \in C\left(X_{0}\right)\left[X_{1}, \ldots, X_{j}\right]$ tel que:

$$
\begin{aligned}
P\left(\left(X_{0}\right)^{q},\left(g\left(X_{0}\right) / a_{1}\right)\left[X_{1}-\alpha_{1}\left(X_{0}\right)\right], \ldots,\left(g\left(X_{0}\right)^{j} / a_{j}\right)\left[X_{j}-\alpha_{j}\left(X_{0}\right)\right]\right) & \\
& =R\left(X_{0}, X_{1}, \ldots, X_{j}\right) P\left(X_{0}, X_{1}, \ldots, X_{j}\right) .
\end{aligned}
$$

La comparaison des termes en $X_{i}(1 \leqslant i \leqslant j)$ prouve qu'en fait $R \in C\left(X_{0}\right)$.

Considérons l'anneau $E=\bigcup_{h \geqslant 1} C\left[X_{0}^{1 / p^{h}}\right]$ et son corps des fractions $U=\bigcup_{h \geqslant 1} C\left(X_{0}^{1 / p^{h}}\right)$. Prenons $Q$ un polynôme de $E\left[X_{1}, \ldots, X_{j}\right]$ de degré total minimal et non nul tel qu'il existe $R\left(X_{0}\right)$ dans $U$ vérifiant:

$$
\begin{aligned}
Q\left(\left(X_{0}\right)^{q},\left(g\left(X_{0} / a_{1}\right)\right)\left[X_{1}-\alpha_{1}\left(X_{0}\right)\right], \ldots,\left(g\left(X_{0}\right)^{j} / a_{j}\right)\left[X_{j}\right.\right. & \left.\left.-\alpha_{j}\left(X_{0}\right)\right]\right) \\
& =R\left(X_{0}\right) Q\left(X_{0}, X_{1}, \ldots, X_{j}\right) .
\end{aligned}
$$

Comme la dérivée de $Q$ par rapport à $X_{i}(1 \leqslant i \leqslant j)$ est de degré total strictement inférieur à celui de $Q$ et vérifie aussi la relation précédente, il s'ensuit que:

$$
Q\left(X_{0}, X_{1}, \ldots, X_{j}\right)=\sum_{1 \leqslant i \leqslant j} c_{i} X_{i}+\sum_{i} d_{i} X_{1}^{p i_{1}} \ldots X_{j}^{p i_{j}}
$$

où $\mathbf{i}=\left(i_{1}, \ldots, i_{j}\right)$ et les $c_{i}, d_{\mathbf{i}}$ sont dans $E$.

L'hypothèse de minimalité sur le degré de $Q$ implique qu'au moins un des $c_{i}$ n'est pas nul, appelons le $c_{i_{0}}$. L'identification des termes en $X_{i_{0}}$ dans l'équation fonctionnelle donne $c_{i_{0}}\left(\left(X_{0}\right)^{q}\right)\left(g\left(X_{0}\right)^{i_{0}} / a_{i_{0}}\right)=R\left(X_{0}\right) c_{i_{0}}\left(X_{0}\right)$. Pareillement si $d_{i}$ est un coefficient d'un terme de degré maximal de $Q$ avec $\mathbf{i}=\left(i_{1}, \ldots, i_{j}\right)$ on a:

$$
d_{\mathrm{i}}\left(\left(X_{0}\right)^{q}\right)\left(g\left(X_{0}\right)^{p\left(i_{1}+2 i_{2}+\ldots+j i_{j}\right)} / b\right)=R\left(X_{0}\right) d_{\mathrm{i}}\left(X_{0}\right),
$$

où $b$ est un élément de $C$.

D'où on obtient l'existence d'un $b^{\prime}$ dans $C$ tel que:

$$
\left(d_{\mathbf{i}} / c_{i_{0}}\right)\left(\left(X_{0}\right)^{q}\right) g\left(X_{0}\right)^{p\left(i_{1}+2 i_{2}+\ldots+j i_{j}\right)-i_{0}} / b^{\prime}=\left(d_{i} / c_{i_{0}}\right)\left(X_{0}\right) .
$$

Comme $i_{0}<p$, l'exposant de $g\left(X_{0}\right)$ dans cette égalité est non nul. Or $d_{\mathbf{i}} / c_{i_{0}}$ est dans $C(X)$ où $X=\left(X_{0}\right)^{1 / p^{m}}$ pour $m$ assez grand, d'après le Lemme 2.3 c'est la fonction nulle. 
Donc un coefficient dominant d'un terme de degré maximal de $Q$ est de la forme $c_{i_{1}}$, si $i_{1} \neq i_{0}$ on a encore une contradiction, $Q$ n'existe pas.

Maintenant un énoncé d'indépendance où on se préoccupe de la dépendance en $\alpha$ de $f(a, \alpha, s)$ :

LEMME 2.5 Soit $s$ un entier naturel non nul. Etant donné $\alpha_{1}(z), \ldots, \alpha_{n}(z), n$ éléments non nuls de $F_{q}(z)$ vérifiant $\operatorname{deg}_{z}\left(\alpha_{i}\right)<\operatorname{seq} /(q-1)(1 \leqslant i \leqslant n), a_{1}, \ldots, a_{n}$, $n$ éléments non nuls de $\bar{k}$, si les fonctions $h_{i}(z):=f\left(a_{i}, \alpha_{i}, s\right)(z)$ sont algébriquement dépendantes sur $C(z)$, alors une combinaison linéaire à coefficients dans $C$ de ces fonctions est une fraction rationnelle de $C(z)$.

PReUve: Le début de la preuve est similaire à celle du lemme précédent. On montre l'énoncé par récurrence sur l'entier $j \leqslant n$. La propriété est vraie pour $j=1$ par le Lemme 2.2. D'après l'hypothèse de récurrence si $h_{1}(z), \ldots, h_{j}(z)$ sont algébriquement dépendantes sur $C(z)$ il existe un polynôme irréductible $P \in C\left[X_{0}, X_{1}, \ldots, X_{j}\right]-C\left[X_{0}\right]$ engendrant l'idéal des relations de dépendances des fonctions sur $C$ tel que pour tout $z$ dans le domaine de convergence on ait:

$$
P\left(z, h_{1}(z), \ldots, h_{j}(z)\right)=0
$$

et donc aussi:

$$
P\left(z^{q},\left(g(z)^{s} / a_{1}\right)\left[h_{1}(z)-\alpha_{1}(z)\right], \ldots,\left(g(z)^{s} / a_{j}\right)\left[h_{j}(z)-\alpha_{j}(z)\right]\right)=0 .
$$

Comme au lemme précédent, on voit qu'il existe $R \in C\left(X_{0}\right)$ tel que:

$$
\begin{aligned}
P\left(\left(X_{0}\right)^{q},\left(g\left(X_{0}\right)^{s} / a_{1}\right)\left[X_{1}-\alpha_{1}\left(X_{0}\right)\right], \ldots,\left(g\left(X_{0}\right)^{s} / a_{j}\right)\left[X_{j}-\alpha_{j}\left(X_{0}\right)\right]\right) & \\
& =R\left(X_{0}\right) P\left(X_{0}, X_{1}, \ldots, X_{j}\right) .
\end{aligned}
$$

Avec les notations $U$ et $E$ du lemme précédent, prenons $Q$ un polynôme de $E\left[X_{1}, \ldots, X_{j}\right]$ de degré total minimal et non nul tel qu'il existe $R\left(X_{0}\right)$ dans $U$ vérifiant:

$$
\begin{aligned}
Q\left(\left(X_{0}\right)^{q},\left(g\left(X_{0}\right)^{s} / a_{1}\right)\left[X_{1}-\alpha_{1}\left(X_{0}\right)\right], \ldots,\left(g\left(X_{0}\right)^{s} / a_{j}\right)\left[X_{j}\right.\right. & \left.\left.-\alpha_{j}\left(X_{0}\right)\right]\right) \\
& =R\left(X_{0}\right) Q\left(X_{0}, X_{1}, \ldots, X_{j}\right) .
\end{aligned}
$$

Il s'ensuit par dérivation que:

$$
Q\left(X_{0}, X_{1}, \ldots, X_{j}\right)=\sum_{1 \leqslant i \leqslant j} c_{i} X_{i}+\sum_{i} d_{i} X_{1}^{p i_{1}}, \ldots, X_{j}^{p i_{j}}
$$

où $\mathrm{i}=\left(i_{1}, \ldots, i_{j}\right)$ et les $c_{i}, d_{\mathrm{i}}$ sont dans $E$.

L'hypothèse de minimalité sur le degré de $Q$ implique qu'au moins un des $c_{i}$ n'est pas nul, appelons le $c_{i_{0}}$. L'identification des termes en $X_{i_{0}}$ dans l'équation fonctionnelle 
donne $c_{i_{0}}\left(\left(X_{0}\right)^{q}\right)\left(g\left(X_{0}\right)^{s} / a_{i_{0}}\right)=R\left(X_{0}\right) c_{i_{0}}\left(X_{0}\right)$. Pareillement si $d_{i}$ est un coefficient d'un terme de degré maximal de $Q$ avec $\mathbf{i}=\left(i_{1}, \ldots, i_{j}\right)$ on a:

$$
d_{\mathrm{i}}\left(\left(X_{0}\right)^{q}\right)\left(g\left(X_{0}\right)^{p s\left(i_{1}+i_{2}+\ldots+i_{j}\right)} / b\right)=R\left(X_{0}\right) d_{\mathbf{i}}\left(X_{0}\right),
$$

où $b$ est un élément de $C$.

D'où on obtient l'existence d'un $b^{\prime}$ dans $C$ tel que:

$$
\left(d_{\mathbf{i}} / c_{i_{0}}\right)\left(\left(X_{0}\right)^{q}\right) g\left(X_{0}\right)^{p s\left(i_{1}+i_{2}+\ldots+i_{j}\right)-s} / b^{\prime}=\left(d_{i} / c_{i_{0}}\right)\left(X_{0}\right),
$$

d'où l'on tire encore $d_{\mathbf{i}}=0$. Donc ici $Q\left(X_{0}, X_{1}, \ldots, X_{j}\right)=\sum_{1 \leqslant i \leqslant j} c_{i} X_{i}+c_{0}$.

Soit $c_{i_{1}}$ un coefficient d'un terme linéaire de $Q$, si $i_{1} \neq i_{0}$, on obtient:

$$
c_{i_{1}}\left(\left(X_{0}\right)^{q}\right)\left(g\left(X_{0}\right)^{s} / a_{i_{1}}\right)=R\left(X_{0}\right) c_{i_{1}}\left(X_{0}\right),
$$

et donc $\left(c_{i_{1}} / c_{i_{0}}\right)\left(\left(X_{0}\right)^{q}\right) a_{i_{0}} / a_{i_{1}}=\left(c_{i_{1}} / c_{i_{0}}\right)\left(X_{0}\right)$ d'où il suit que $c_{i_{1}} / c_{i_{0}}$ est un élément de $C$, ce qui implique le résultat attendu.

\section{INDÉPENDANCE DES NOMBRES}

Dans ce paragraphe, on va utiliser l'analogue du théorème d'indépendance algébrique de Becker [1] sur la méthode de Mahler qu'on a établi dans [7]. Plus spécialement rappelons l'énoncé du Théorème 2 de [7] (ou de [8]) dans le cas $T(z)=z^{q}$ et avec le changement de variable de $z$ en $1 / z$.

THÉOṘ̀M 3.1. (Voir [7] ou [8].) Soit $f_{1}, \ldots, f_{m}$ des fonctions de $C$ dans $C$ ayant un développement de Taylor convergeant dans un domaine $|z|>r \geqslant 1$ et à coefficients dans une extension finie $k_{1}$ de $k$. Supposons que ces fonctions sont algébriquement indépendantes sur $C(z)$ et qu'il existe des fractions rationnelles $a_{i}(z)$ et $b_{i}(z)(1 \leqslant i \leqslant m)$ dans $k_{1}(z)$ telles que:

$$
f_{i}(z)=a_{i}(z) f_{i}\left(z^{q}\right)+b_{i}(z) \quad(1 \leqslant i \leqslant m) .
$$

Alors pour tout $\gamma$ algébrique non nul tel que $|\gamma|>r$, tel pour tout entier $n, \gamma^{q^{n}}$ n'est zéro d'aucun $a_{i}(z)$ et pôle d'aucun $b_{i}(z)$, les nombres. $f_{1}(\gamma), \ldots, f_{m}(\gamma)$ sont algébriquement indépendants sur $k$.

Preuve du ThÉorìme 1.3: Avec les notations du paragraphe 2, on choisit $g(z)$ $=z^{q}-T$ et pour $1 \leqslant s \leqslant p-1$, on considère $f_{s}(z)=f\left((-1)^{s}, 1, s\right)(z)$. D'après le Lemme 2.4, ces fontions sont algébriquement indépendantes sur $C(z)$.

La relation $\zeta(s)=\sum_{0 \leqslant h \leqslant \infty}\left((-1)^{h s}\right) /\left(\left(L_{h}\right)^{s}\right)$ (voir [3]) montre qu'on a $f_{s}(T)$ $=\zeta(s)$. Les conditions du théorème précédent sont vérifiées et on a donc prouvé le Théorème 1.3. 
Preuve du Théorème 1.4: Ici on laisse $g(z)=z^{q e}-T$. Si $\alpha$ est un élément de $F_{q}\left(T^{1 / e}\right)$, on notera $\alpha(z)$ l'élément de $F_{q}(z)$ obtenu en remplaçant formellement $T^{1 / e}$ par $z$ dans l'expression de $\alpha$. Notons que si le degré dans $C$ de $\alpha$ est $<q /(q-1)$, le degré en $z$ de $\alpha(z)$ est $<e q /(q-1)$. Donc si $\alpha_{1}, \ldots, \alpha_{n}$ sont des éléments de $F_{q}\left(T^{1 / e}\right)$ de degré $<q /(q-1)$, on peut considérer $f_{i}(z)=f\left(-1, \alpha_{i}(z), 1\right)(z)(1 \leqslant i \leqslant n)$. D'après l'expression du logarithme donnée au paragraphe 1 , on a alors $f_{i}\left(T^{1 / e}\right)=\log _{\phi}\left(\alpha_{i}\right)$. Si on montre l'indépendance algébrique des fonctions $f_{i}(z)(1 \leqslant i \leqslant n)$, on concluera encore à l'aide du théorème ci-dessus à l'indépendance algébrique des logarithmes.

D'après le Lemme 2.5 si ces fonctions sont dépendantes, une combinaison linéaire non triviale à coefficients dans $C$ de ces fonctions est dans $C(z)$. Or ces fonctions ont un développement de Taylor à coefficients rationnels donc s'il existe une combinaison linéaire à coefficients dans $C$ qui soit dans $C(z)$, il en existe une à coefficients dans $A$ appartenant à $k(z)$. En effet, par spécialisation il y en a une algébrique, on élève à la puissance $p^{f}$ ( $f$ suffisamment grand) pour la rendre séparable, comme il y a un élément de trace non nulle dans chaque extension séparable, le fait de prendre la trace amène à une combinaison dans $A$ non triviale entre les puissances $p^{f}$ des fonctions. Choisissant $f$ minimal, puis le degré en $T$ de la relation également minimal, on déduit par dérivation par rapport à $T$ que $f=0$.

Notons cette combinaison non triviale à coefficients dans $A$ :

$b_{1} f_{1}(z)+\cdots+b_{n} f_{n}(z)=b(z)$. Comme toutes les séries sont convergentes en $T^{1 / e}$, il s'ensuit la relation $b_{1} \log _{\phi}\left(\alpha_{1}\right)+\ldots+b_{n} \log _{\phi}\left(\alpha_{n}\right)=b\left(T^{1 / e}\right)$. Prenant I'exponentielle des deux termes on a que $e_{\phi}\left(b\left(T^{1 / e}\right)\right)$ est algébrique donc $b$ est nulle (théorème de Wade [12]) et d'après l'hypothèse d'indépendance linéaire des logarithmes, tous les $b_{i}$ sont nuls, ce qui achève la preuve du Théorème 1.4 (notons qu'en fait la preuve donne aussi une nouvelle démonstration du théorème de Baker dans le cas rationnel).

La méthode ne semble pas s'appliquer aux éléments $\alpha$ de $F_{r}\left(T^{1 / e}\right)$ quand $F_{r}$ est une extension finie stricte de $F_{q}$. Néanmoins on a aussi le résultat suivant:

ThÉORÈM 3.3. Soit $\varepsilon$ un élément de $F_{q}-\{0,1\}$ et $\mu$ une solution de l'équation $X^{q-1}=1 / \varepsilon$ alors $\log _{\phi}(1)$ et $\log _{\phi}(\mu)$ sont algébriquement indépendants sur $k$.

Preuve: Par hypothèse $\mu$ n'est pas dans $F_{q}$ et pour tout entier $h, \mu^{q^{h}-1}=\varepsilon^{-h}$. On considère alors les deux fonctions $f_{1}(z)=f(-1,1,1)(z)$ et $f_{2}(z)=f(-1 / \varepsilon, 1,1)(z)$ avec $g(z)=z^{q}-T$. D'après le Lemme 2.5, si ces fonctions sont algébriquement dépendantes sur $C(z)$, il existe une relation $b_{1} f_{1}(z)+b_{2} f_{2}(z)=b(z)$ où $b_{1}, b_{2}$ sont dans $k$ et $b(z)$ est dans $k(z)$ d'où l'on tire $b_{1} \log _{\phi}(1)+b_{2} \log _{\phi}(\mu) / \mu=b(T)$.

D'après le théorème de Baker, il suffit alors de prouver que $\log _{\phi}(1)$ et $\log _{\phi}(\mu)$ sont linéairement indépendants sur $k$. Si $c$ et $d$ sont dans $F_{q}[T]-\{0\}$ et vérifient $c \log _{\phi}(1)$ $=d \log _{\phi}(\mu)$ alors en prenant l'image par l'exponentielle on obtient $\phi(c)(1)=\phi(d)(\mu)$ ce qui est absurde car le terme de droite n'est pas dans $F_{q}[T]$.

Enfin notons qu'on peut aussi comparer $\pi$ et les logarithmes d'éléments de $k$ : 
ThÉorèm 3.4. Soit $\alpha$ est un élément de $F_{q}(T)$ non nul et de degré $<q /(q-1)$. Alors $\pi$ et $\log _{\phi}(\alpha)$ sont algébriquement indépendants sur $k$ dans chacun des cas suivants et seulement dans ces cas:

(a) $p>2$;

(b) $q=2$ et $\alpha$ n'est pas un polynôme de degré $\leqslant 1$;

(c) $q>p=2$ et $\alpha$ n'appartient pas à $F_{q}$.

Preuve: On prend $g(z)=z^{q}-T$ et comme dans la preuve du Théorème 1.4, $f_{1}(z)=f(-1, \alpha(z), 1)(z)$ puis $f_{2}(z)=f(1,1, p-1)(z)$. Si $p$ est différent de 2 , alors le Lemme 2.4 donne l'indépendance algébrique des deux fonctions et la spécialisation en $z=T$ fournit le résultat puisque $\zeta(p-1) / \pi^{p-1}$ est rationnel.

Si $p=2$, et $\alpha$ de degré $<2$, il s'agit en fait de prouver l'indépendance algébrique de $\log _{\phi}(\alpha)$ et $\log _{\phi}(1)$. D'après le Théorème 1.4, il s'agit de prouver leur indépendance linéaire sur $\dot{F}_{q}[T]$. Si $c$ et $d$ sont dans $F_{q}[T]-\{0\}$ et vérifient $c \log _{\phi}(1)=d \log _{\phi}(\alpha)$, on a alors $\Phi(c)(1)=\Phi(d)(\alpha)$. Comme le terme de droite de cette dernière égalité est dans $F_{q}[T]$ et que $\phi(d)(X)$ est de coefficient dominant dans $F_{q}$, il suit que $\alpha$ appartient à $F_{q}[T]$. Il ne reste alors que le cas où le degré de $\alpha$ est 1 . Quitte à le prendre unitaire, on peut écrire $\alpha=T+x$ où $x$ est dans $F_{q}$. Il vient alors $\Phi(d)(T)=\Phi(d-c x)(1)$, si $q=2$ on prend $d=T=c x$. Si $q$ est différent de 2, l'examen du degré en $T$ dans l'équation précédente montre qu'elle n'a pas de solution.

REmarques. (a) Ce résultat améliore le Théorème 6 de [6] où on obtenait la transcendance de quotients d'un logarithme par des puissances de $\pi$. Notons que pour $p=2$, ces résultats sur la transcendance de quotients n'étaient pas non plus démontrés y compris par les méthodes automatiques de [2].

(b) Après que ce texte fut rédigé nous avons appris que Matthew A. Papanikolas [11] avait obtenu l'indépendance algébrique des logarithmes d'éléments algébriques étendant ainsi au cas général (pour le module de Carlitz) les résultats de notre Théorème 1.4. Sa méthode repose sur un développement du formalisme des catégories tannakiennes dans le cadre des $t$-modules et $t$-motifs comme signalé dans notre introduction (voir aussi [ABD] pour les fondements de ce travail). Il est intéressant de remarquer à ce propos qu'à l'heure actuelle aucune des deux méthodes (ni celle de Mahler ni celle des $t$-motifs) ne permet d'obtenir des résultats d'indépendance algébrique satisfaisants en rang supérieur à 2 .

\section{REFERENCES}

[1] P.G. Becker, 'Algebraic independence of the values of certain series by Mahler's method', Monatsh. Math. 114 (1992), 183-198.

[2] V. Berthé, 'Automates et valeurs de transcendance du logarithme de Carlitz', Acta Arith. 66 (1994), 369-390. 
[3] L. Carlitz, 'On certain functions connected with polynomials in a Galois field', Duke Math. J. 1 (1935), 137-168.

[4] L. Denis, 'Théorème de Baker et modules de Drinfeld', J. Number Theory 43 (1993), 203-215.

[5] L. Denis, 'Dérivées d'un module de Drinfeld et transcendance', Duke Math. J. 80 (1995), 1-13.

[6] L. Denis, 'Méthodes fonctionnelles pour la transcendance en caractéristique finie', Bull. Austral. Math. Soc. 50 (1994), 273-286.

[7] L. Denis, 'Indépendance algébrique des dérivées d'une période du module de Carlitz', $J$. A ustral. Math. Soc. 69 (2000), 8-18.

[8] L. Denis, 'Indépendance algébrique de différents $\pi$ ', C.R. Acad. Sci. Paris, Sér. 1 Math. 327 (1998), 711-714.

[9] G. Damamme et Y. Hellegouarch, 'Transcendence of the values of the Carlitz Zeta function by Wade's method', J. Number Theory 39 (1991), 257-278.

[10] B. de Mathan, 'Un critère de transcendance en caractéristique positive', C.R. Acad. Sci. Paris, Sér. 1 Math. 319 (1994), 427-432.

[11] M.A. Papanikolas, 'Tannakian duality for Anderson-Drinfeld motives and algebraic independence of Carlitz logarithms', (preprint 2005).

[12] L.I. Wade, 'Certain quantities transcendental over $G F\left(p^{n}, x\right)$ ', Duke Math. J. 8 (1941), 701-720.

[13] J. Yu, 'Analytic homomorphism into Drinfeld modules', Ann. of Math. (2) 145 (1997), 215-233.

[14] J. Yu, 'Transcendance and Drinfeld modules : Several variables', Duke Math. J. 58 (1989), 559-575.

Laboratoire Paul Painlevé UMR CNRS 8524

U.F.R. de Mathématiques Pures et Appliquées, Bât. M2

Université des Sciences et Technologies de Lille 1

59665 Villeneuve d'Ascq Cedex

France

e-mail: Laurent.Denis@univ-lille1.fr 